\title{
Didaktik der Geographie - Wissenschaft aus eigenem Recht oder Anhängsel der Geographie?*
}

Welchen Namen man der hier zur Diskussion stehenden Disziplin auch immer gibt - «Didaktik der Geographie», «Geographiedidaktik», «Fachdidaktik Geographie»: aus jedem hört man heraus, die Didaktik der Geographie sei eine Funktion der Geographie, eine von der Geographie abhängige Größe also, und verdanke ihre Existenz allein der Existenz der Geographie. Überspitzt ausgedrückt: die Geographiedidaktik sei ein «Anhängsel» der Geographie, wovor SCHRAND (1981, S. 65) warnt, bzw. sie fungiere «weithin als verlängerter Arm der Fachwissenschaft, als Agentur für die Propaganda des fachwissenschaftlichen Welt- und Selbstbildes, als Transformation von Fachwissenschaft in Unterrichtseinheiten», wie HARD (1979, S. 35/36) es als tatsächlich gegeben ansieht. Ähnlich muß es wohl auch mit anderen bzw. letztlich allen Fachdidaktiken stehen, wie man aus Etikettierungen wie «Verkaufstechnik» oder «nachträgliche(s) Anspritzverfahren» (vgl. dazu OTTO 1984, S. 31, 34; PÖPPEL 1987, S. 21) u. ä. m. schließen muß.

Ist diese Sicht der Beziehung zwischen Geographiedidaktik und Geographie nun begründet und damit richtig? Ist die Geographiedidaktik also ein Anhängsel der Geographie, oder ist sie eine Wissenschaft aus eigenem Recht? Aus meiner Sicht, wie wohl auch aus der Sicht zumindest derjenigen Kollegen, die die Geographiedidaktik nicht für eine Subdisziplin der Geographie halten, ist sie selbstverständlich eine Wissenschaft aus eigenem Recht! Inwiefern? Das will ich nachfolgend aus verschiedenen Blickwinkeln begründen.

\section{Wissenschaftstheoretische Begründung}

Der erste Begründungsansatz ist methodologischer Art. Aus eigenem Recht besteht eine Wissenschaft hiernach dann, wenn sie die für Wissenschaft als Forschung (Wissenschaft als Lehre lasse ich im folgenden aus naheliegenden Gründen außer Acht) konstitutiven Kriterien erfüllt.

\footnotetext{
* Überarbeitete und gekürzte Fassung eines Vortrages, gehalten am 3.7.1989 im Rahmen des Geographischen Kolloquiums der Universität Osnabrück.
}

\subsection{Kriterien für Wissenschaft(lichkeit)}

$\mathrm{Zu}$ diesen gehört bekanntlich zunächst der Gegenstand (vgl. MENNE 1980, S. 7), in empirischen Wissenschaften «Realobjekt» genannt.

$\mathrm{Da}$ Gegenstände, in Abwandlung eines entsprechenden Wortes von POPPER ( ${ }^{2} 1984$, S. 225), aber nicht antworten, wenn sie nicht gefragt werden, bzw. da die Gegenstände von Wissenschaft überhaupt erst durch entsprechende Fragen generiert werden (vgl. HOLZKAMP 1968, S. 43, 44), bedarf es weiterhin einer disziplinkonstituierenden bzw. -leitenden Fragestellung, die dann den Status eines disziplinären Paradigmas hat und üblicherweise «Erkenntnisobjekt» genannt wird. Falls derselbe Gegenstand dann zu verschiedenen solcher paradigmatischen Fragestellungen Anlaß gibt - und das ist ja die Regel -, ist er zugleich Forschungsobjekt mehrerer Wissenschaften.

Mithin wird eine Wissenschaft letztlich erst durch die Fragestellung bzw. die Verknüpfung ihrer Fragestellung mit ihrem bzw. einem Gegenstand definierbar, abgrenzbar und identifizierbar (WEICHHART 1975, S. 70). Allerdings gilt dies nach WEINGARTNER $(21978$, S. 130, 212) nur auf der Ebene der abhängigen Variablen, der jeweils $z u$ erklärenden bzw. $z u$ begründenden Sätze also. Auf der Ebene der unabhängigen Variablen dagegen, der jeweils erklärenden bzw. begründenden Sätze also, verarbeitet jede Wissenschaft außer eigenen Aussagen auch solche anderer jeweils in Frage kommender Wissenschaften, so daß eine interdisziplinäre Trennschärfe hier nicht mehr gegeben ist.

Über Gegenstand und Fragestellung hinaus ist drittens jedoch noch zu fordern, daß eine Disziplin, die sich als Wissenschaft versteht, einerseits natürlich überhaupt Erkenntnisse produziert und diese in entsprechenden Sätzen bzw. Aussagen formuliert. Da dies aber auch im Alltag geschieht, ist dieser Erkenntnisgewinn nur dann wissenschaftlich, wenn er andererseits auf systematische, intersubjektiv überprüfbare und methodisch-methodologisch normierte Weise zustande kommt und seine Ergebnisse überdies in normierter Terminologie allgemein zugänglich

\footnotetext{
Helmuth Köck, Prof. Dr., Universität Hildesheim
} Marienburger Platz 22, D-3200 Hildesheim. 
und damit überprüfbar gemacht werden (vgl. MENNE 1980, S. 7-9; SEIFFERT 101983, I, S. 84-88; WEINGARTNER 21978, S. 43-129).

\subsection{Zur Erfüllung dieser Kriterien durch die Geographiedidaktik}

Erfüllt die Geographiedidaktik nun diese drei Kriterien?

\section{- Gegenstand:}

Betrachten wir zunächst den Gegenstand der Geographiedidaktik. Traditionell ist dies der Geographieunterricht an allgemeinbildenden Schulen. Allerdings stellt dieser lediglich einen, wenngleich den weitaus bedeutendsten Gegenstandsbereich der Geographiedidaktik dar. Weitere, an Bedeutung zunehmende Gegenstandsbereiche werden in jüngerer Zeit im Geographieunterricht an Berufssowie Sonderschulen, in der geographischen Erwachsenenbildung an Volkshochschulen, im Geographiestudium an Hochschulen und Universitäten usw. gesehen (vgl. EBINGER 1971, S. 21; KÖCK 1986.1, S. 24; 1986.2, S. 115/116; SCHREIBER 1981, S. 16).

All diese und weitere Gegenstandsbereiche der Geographiedidaktik bestehen unabhängig von der Existenz der geographischen Wissenschaft und sind aus ihr auch nicht ableitbar. Die geographische Unterweisung findet in den genannten Bereichen nicht statt, weil es die Geographie bzw. das geographische Wissen nun einmal gibt, sondern weil irgendwelche Zwecke dies jeweils erfordern (vgl. auch RICHTER et al. 1971, S. 147).

Und selbst wenn es die geographische Wissenschaft nicht gäbe, könnte es gleichwohl die genannten Formen geographischer Wissensvermittlung geben (vgl. KÖCK 1986.3, S. 139/140; 1989, S. 11): Denn der empirische Gegenstand dieser Unterrichtsveranstaltungen besteht bzw. bestand ja unabhängig von der Existenz der Geographie; desgleichen sind die Gründe, weshalb in den betreffenden Veranstaltungen geographisches Wissen vermittelt wird, unabhängig von der Geographie. Allenfalls stellte sich dann die Frage, wer das zu vermittelnde Wissen über den Gegenstand Erde überhaupt erarbeitet und bereitstellt. Lediglich insoweit also entstünde dann bzw. besteht eine Abhängigkeit von der geographischen Wissenschaft.

Nun hat diese Gegenstandsbestimmung aber noch reichlich additiven Charakter. Man müßte, um den Gegenstand der Geographiedidaktik in dieser Weise umfassend zu bestimmen, ja sämtliche Bereiche, in denen geographische Erkenntnisse gelehrt und gelernt werden, aufzählen, was offenkundig reichlich umständlich wäre. Versucht man dagegen, den Gegenstand der Geographiedidaktik durch einen einzigen Begriff zu erfassen, so könnte man ihn wie folgt definieren: Gegenstand der Geographiedidaktik ist das institutionalisierte Lehren und Ler- nen geographischer Aussagen (кӧск 1986.1, S. 23/ $24 ; 1986.2$, S. 114). Soweit keine ausdrückliche Einschränkung gemacht wird, schließt dies, analog zum Gegenstand der Didaktik überhaupt (vgl. z. B. BÖNSCH 1985, S. 6; SCHMIEL 1978, S. 23/24; STEUBER 1981, S. 113, 126, 136), sämtliche Arten von Institutionen ein, in denen Geographie gelehrt und gelernt wird. Zugleich grenzt der Terminus «institutionalisiert» den Gegenstand der Geographiedidaktik gegen jene Bereiche $a b$, in denen geographisches Wissen informell und ohne didaktische Fundierung/Anleitung weitergegeben bzw. angeeignet wird, wie beispielsweise in der Familie im Rahmen der Primärsozialisation.

Nun zeigt sich aber, daß der so definierte Gegenstand der Geographiedidaktik methodologisch aus zwei Komponenten besteht, nämlich aus einem Gegenstand im engeren und eigentlichen Sinn und einer Fragestellung. Der Gegenstand im engeren und eigentlichen Sinn wird dabei durch die Menge der geographischen Aussagen gebildet. Demgegenüber richtet sich die Fragestellung in erster Annäherung auf die Zwecke und Mittel des instituionaliserten Lehrens und Lernens geographischer Aussagen.

Wenn der eigentliche Gegenstand der Geographiedidaktik somit aber durch die Menge der geographischen Aussagen gebildet wird: entsteht dadurch dann nicht eine Abhängigkeitsbeziehung der Art, daß die Geographiedidaktik doch zum Anhängsel der Geographie wird? Wenngleich dies aus pragmatischer Sicht so scheint, so ist dies bei methodologischer Betrachtung jedoch gleichwohl nicht der Fall. Denn keine Wissenschaft muß sich ihren Gegenstand als solchen erst noch schaffen, was natürlich nicht ausschließt, daß sich eine Wissenschaft auch mit ihren nach und nach erzeugten eigenen Produkten wissenschaftlich auseinandersetzt und dies im Rahmen der intersubjektiven Überprüfung ja auch muß. Vielmehr existieren die Gegenstände der einzelnen Wissenschaften unabhängig von diesen als empirische Substrate oder mentale Erzeugnisse, wobei es gleichgültig ist, welchen Ursprungs diese jeweils sind. Ob ein irgendwie gegebener Gegenstand aber Gegenstand von Wissenschaft wird, hängt - wie bereits in 1.1 bemerkt - nicht von seiner bloßen Existenz ab, sondern davon, ob er zum Ziel paradigmatischer Fragestellung und wissenschaftlicher Untersuchungsweisen wird. Bleiben entsprechende Fragestellungen und Untersuchungen dagegen aus, so hat der betreffende Gegenstand auch nicht den methodologischen Status, Gegenstand einer oder mehrerer Wissenschaften zu sein.

In diesem Sinne kann die Menge der geographischen Aussagen als mental erzeugtes Substrat betrachtet werden, das als solches und zunächst nichts tut als lediglich da zu sein. Ob und wofür es ggf. verwendet wird, ist von ihm selbst völlig unabhängig. Auch ob es zum Gegenstand wiederum anderer Wissenschaften wird, hängt nicht von ihm 
selbst ab, sondern davon, ob eine entsprechende Fragestellung an es herangetragen und auf wissenschaftliche Weise untersucht wird. Entsprechend auch wird die Menge der geographischen Aussagen nur dadurch zum Gegenstand der Geographiedidaktik, daß die didaktische Fragestellung an sie herangetragen und auf wissenschaftliche Weise untersucht wird. Würde dies nicht geschehen bzw. geschehen sein, hätte der geographische Erkenntnisbestand auch nicht den methodologischen Status, Gegenstand der Wissenschaft Geographiedidaktik zu sein bzw. gewesen zu sein.

\section{- Fragestellung:}

Betrachtet man nun die Fragestellung der Geographiedidaktik, so ist diese von derjenigen der Geographie kategorial verschieden und logisch ebenfalls unabhängig. Denn während die Geographie nach der räumlichen Ordnung geosphärischer Sachverhalte fragt, geht es der Geographiedidaktik um das Lehren und Lernen von Aussagen bzw. Erkenntnissen über die räumliche Ordnung geosphärischer Sachverhalte, um das Lehren und Lernen geographischer Aussagen also. In operationalisierter Form lautet die geographiedidaktische Fragestellung dann: Wem bzw. von wem werden warum/wozu welche geographischen Aussagen wie und mit welchem Erfolg gelehrt bzw. gelernt (vgl. KöcK 1986.1, S. 25; ${ }^{21986, ~ S . ~ 116 / 117) . ~ W e d e r ~ d a ß ~ u ̈ b e r h a u p t ~ u n d ~}$ wozu noch welche noch wie geographische Aussagen gelehrt und gelernt werden bzw. werden sollen, läßt sich allein aus der Existenz der Geographie als Wissenschaft oder der Menge der geographischen Aussagen ableiten. Auch da $B$ die existierenden geographischen Aussagen unter genau dieser Fragestellung Gegenstand von Forschung sind bzw. werden, ist weder durch die Geographie noch durch deren Aussagensystem impliziert. Es handelt sich bei der Fragestellung der Geographiedidaktik eben um ein didaktisches und nicht um das geographische Paradigma, das von diesem eben deshalb kategorial verschieden und ihm sozusagen aufgesetzt ist. Somit aber ist evident, daß die Geographiedidaktik auch von ihrer Fragestellung her kein Anhängsel der Geographie, sondern eine logisch unabhängig von dieser bestehende eigenständige Disziplin ist.

Allenfalls insofern könnte man eine Abhängigkeit erkennen, als die Geographiedidaktik zwar geographische Aussagen zum Gegenstand hat, die Definition dessen, was geographisch ist, aber Sache der Geographie ist. Jedoch ist die Geographiedidaktik auch in dieser Hinsicht nicht auf die Existenz der Geographie bzw. der von dieser definierten geographischen Fragestellung angewiesen. Denn $d a \beta$ die geosphärische Welt zumindest in der Schule auch in ihrer räumlichen Dimension erschlossen werden soll, begründet sich unabhängig von der Existenz eines dementsprechenden wissenschaftlichen Paradigmas (vgl. KÖCK 1989, S. 11). Daß man diese räumliche Dimension dabei im Sinne geosphärischer Lagebziehungen versteht, erfordert gleichfalls nicht notwendig die Existenz eines dementsprechenden geographischen Paradigmas bzw. einer daran orientierten Geographie. Denn Räumlichkeit als Kategorie erdbezogenen Erkennens und Verhaltens ist eine evolutionär gewordene und somit ubiquitär vorhandene Kategorie (vgl. RIEDL 21980 , S. $76,181 / 182$; 1985, S. 22 ; VOLLMER 1975 , S. 127 , 129), so daß sie nicht erst durch eine entsprechende Wissenschaft entdeckt werden braucht. $\mathrm{Da}$ es nun aber eine Wissenschaft, eben die Geographie, gibt, die die räumliche Beschaffenheit der Geosphäre zum Erkenntnisobjekt hat, belegt vor diesem Hintergrund zunächst nur den qualitativen Sprung von der alltäglichen zur wissenschaftlichen Weltsicht, wenngleich es tatsächlich natürlich mehr als nur dies bedeutet.

\section{- Vorgehensweise und Terminologie:}

Über den eigenständigen Gegenstand sowie die eigenständige Fragestellung hinaus hatte ich aus methodologischer Sicht drittens noch gefordert, daß eine Disziplin, die sich als Wissenschaft versteht, nun natürlich auch Erkenntnisse produzieren und in entsprechenden Aussagen/Sätzen formulieren muß, und zwar auf wissenschaftsgemäße Weise. Daß die Geographiedidaktik nun aber tatsächlich Aussagen produziert, ist wohl evident. Evident dürfte auch sein, da $\beta$ diese Aussagen nicht von der Geographie mitproduziert werden bzw. werden können, es sei denn, ein Geograph ist zugleich auch Geographiedidaktiker im hier entfalteten Sinn und bezeichnet sich auch entsprechend. Weiterhin dürfte evident sein, daß die Geographiedidaktik diese Aussagen, zumindest prinzipiell, auf wissenschaftliche Weise im eingangs definierten Sinn produziert, wobei, soweit die Geographiedidaktik auch normative und empirisch-normative (technologische) Aussagen formuliert, der Grad der Wissenschaftlichkeit allerdings notwendig unter $100 \%$ bleibt. Freilich hat die Geographie, was meistens übersehen wird, diesbezüglich dieselben Probleme, jedenfalls soweit sie, etwa im Kontext der Angewandten Geographie, auch normative und technologische raumbezogene Aussagen formuliert (vgl. KÖCK 1988.2).

Wie gut die Anforderungen an Wissenschaftlichkeit dabei tatsächlich erfüllt werden, steht auf einem anderen Blatt. Doch sollte man der Geographiedidaktik hierbei ihr noch junges Alter und die damit verknüpfte Entwicklungsnotwendigkeit und Entwicklungsfähigkeit zugute halten und vor allem nicht vergessen, daß auch die Geographie einmal eine junge Disziplin war. Wenn die Geographiedidaktik somit auch das dritte genannte methodologische Kriterium, zumindest im Prinzip, erfüllt, dann kann aus methodologischer Sicht festgehalten werden, daß die Geographiedidaktik eine Wissenschaft aus eigenem Recht und nicht Anhängsel der Geographie ist. 


\section{Wissenschaftssystematische Begründung}

Von dieser methodologischen komme ich nun zur wissenschaftssystematischen Betrachtung des Verhältnisses von Geographie und Geographiedidaktik. Bekanntlich wird bzw. zumindest wurde hierzu selbst in der Geographiedidaktik der Standpunkt vertreten, die Geographiedidaktik sei eine Subdisziplin der Geographie, so etwa von BAUER (1974, S. 26), JAHN (1974, S. 62), SPERLING (1969, S. 86). Jedoch ist dieser Standpunkt unrichtig. Denn maßgeblich für die Abgrenzung und Zuordnung von Wissenschaften sind nach WEINGARTNER, wie bereits erwähnt, deren abhängige Variablen, also deren $z u$ erklärende bzw. $z u$ begründende Aussagen. Diese aber haben in der Geographiedidaktik nicht die räumliche Ordnung der Geosphäre zum Gegenstand, sondern das institutionalisierte Lehren und Lernen von Aussagen über die räumliche Ordnung der Geosphäre, stellen also keine geographischen Aussagen, sondern Aussagen über geographische Aussagen dar. Folgendes Aussagenpaar mag dies verdeutlichen:

1) Das Klima der Erde ist hauptsächlich zonal geordnet.

2) Mit Hilfe von Modellen erkennen die Schüler, daß das Klima der Erde hauptsächlich zonal geordnet ist.

Während Satz 1) ein Merkmal der erdräumlichen Ordnung zur abhängigen Variablen hat, das seinerseits nun durch entsprechende unabhängige Variablen erklärt werden müßte, mithin also eindeutig ein geographischer Satz ist, hat Satz 2) eine These darüber zur abhängigen Variablen, wie die Schüler die zonale Ordnung des Klimas erkennen können. Hierbei wäre dann zu begründen, weshalb gerade Modelle zur Erkenntnis der geozonalen Raumstruktur des Klimas geeignet sind, so daß dieser Satz eindeutig ein geographiedidaktischer Satz ist. Mithin also sind geographiedidaktische Aussagen logisch grundverschieden von geographischen Aussagen; sie sind diesen nicht sozusagen «blutsverwandt», sondern allenfalls «anverwandt». Wenn sich die abhängigen Variablen der Geographiedidaktik in logischer Hinsicht somit aber so grundsätzlich von denen der Geographie unterscheiden, dann kann die Geographiedidaktik keine Subdisziplin und erst recht kein Anhängsel der Geographie sein. Vielmehr hat sie auch aus dieser Sicht den Status einer gegenüber der.Geographie eigenständigen Wissenschaft.

Wohl allerdings hat die Geographiedidaktik gegenüber der Didaktik den Status einer Subdisziplin. Denn wenn die Didaktik die Wissenschaft vom institutionalisierten Lehren und Lernen überhaupt ist (STEUBER 1981, S. 113, 126, 136), und wenn, wie vorhin definiert, die Geographiedidaktik die Wissenschaft vom institutionalisierten Lehren und Lernen geographischer Aussagen ist (vgl. auch EBINGER
1971, S. 9; HARD 21975, S. 402), «dann stellt die Didaktik einen Gattungsbegriff und die Geographiedidaktik einen diesem untergeordneten Artbegriff dar. Mithin ist die Geographiedidaktik aus begriffslogischer wie wissenschaftssystematischer Sicht eine Subdisziplin der Didaktik» (KÖCK 21990 ; auch 1986.1, S. 30/31; ${ }^{2} 1986$, S. 126/127; 11990). Ähnlich sehen dies auch BIRKENHAUER (1975, S. 162-164; 1977, S. $153-154 ; 1980$, S. 18; 1986, S. 211), EBINGER (1971, S. 51; 1977, S. 169/170), HARD ( 21975 , S. 403/ 404), OBLINGER (1974, S. 95) sowie partiell auch SCHRAND (1989, S. 7). Allerdings ist diese Unterordnung unter die Didaktik nur mittelbarer Art. Unmittelbar gehört die Geographiedidaktik dagegen dem Block der Fach- bzw. Sachgebietsdidaktiken, im Unterschied zur Allgemeinen Didaktik, an.

Dabei hat der Terminus «Fachdidaktik» auf dieser allgemein- bzw. artbegrifflichen Ebene seine Berechtigung, insofern er hier die Unterscheidung gegenüber der «Allgemeinen Didaktik» ausdrückt. Auf der individualbegrifflichen Ebene ist er dagegen deplaziert, weil partiell tautologisch und tendenziell diskriminierend: tautologisch insofern, als die Didaktik der Geographie bzw. Geographiedidaktik, im Unterschied zur Allgemeinen Didaktik, per se Fach- bzw. Sachgebietsdidaktik ist und des Präfixes «Fach-» daher nicht mehr bedarf; diskriminierend insofern, als das diesbezügliche Begriffspaar «Fachdidaktik Geographie - Fachwissenschaft Geographie» impliziert und suggeriert, die Didaktik der Geographie sei, im Unterschied zur Fachwissenschaft Geographie, keine Wissenschaft. Die korrekte Bezeichnung kann auf der individualbegrifflichen Ebene nur «Didaktik der Geographie» oder «Geographiedidaktik» lauten, wobei letztlich dem Terminus «Geographiedidaktik» der Vorzug zu geben ist, da dieser weniger die Disziplin- als vielmehr die Sachorientierung assoziiert: Es wird ja nicht «Geographie» bzw. «die Geographie», zudem in systematischer Vollständigkeit, gelehrt und gelernt, sondern "Geographisches» in zudem didaktisch begründeter Auswahl.

\section{Wissenschaftsorganisatorische Begründung}

Vor dem Hintergrund dieser wissenschaftssystematischen Ausführungen bietet es sich an, kurz auf die wissenschaftsorganisatorische Stellung der Geographiedidaktik einzugehen. Denn wenn die Geographiedidaktik irgendwo tatsächlich die Stellung eines Anhängsels hat, dann am ehesten auf dieser Ebene.

Speziell dort wird dies offenkundig, wo die Geographiedidaktik in geographische Institute integriert ist oder von denselben Personen zusammen mit der Geographie vertreten wird. Im letzteren Fall bringt es alleine schon der Dienstauftrag mit sich, daß die 
Geographiedidaktik zumindest in der Lehre gegenüber der Geographie zu kurz kommt. In der Forschung dagegen muß dies nicht sein; jedoch ergibt sich dies vielfach mit einer gewissen Zwangsläufigkeit. Wo die Geographiedidaktik dagegen geographischen Instituten zugeordnet ist, ist sie in der Lehre zumindest im Prinzip ungeschmälert, sofern die betreffenden Kollegen nicht aus irgendwelchen Gründen, darunter vielleicht auch Imagegründen, auch geographische Lehrveranstaltungen abhalten. In der Forschung dagegen führt die Geographiedidaktik bei dieser Konstellation vielfach zwangsläufig ein Schattendasein: Denn angesichts der tiefsitzenden, wenngleich, wie ich 1988.2 gezeigt habe, zumindest methodologisch unbegründeten Vorurteile und verbreiteten Geringschätzung der meisten Geographen der Geographiedidaktik gegenüber (vgl. auch SCHRAND 1989, S. 5) ist der Profilierungszwang nahezu unausweichlich. Und da Profilierung den Kollegen von der Geographie gegenüber durch geographiedidaktische Arbeiten immer noch einer Sisyphusarbeit gleichkommt, weicht man zwangsläufig auf geographische Forschung aus. Das bedeutet dann aber, daß die Geographiedidaktik selbst bei Geographiedidaktikern zu einem Anhängsel der Geographie wird.

Statt diese leidige Imageproblematik, derjenigen der Geographie vor allem in ihrer Frühzeit durchaus nicht unähnlich (vgl. HARD 1979; SCHRAND 1983), hier weiter zu vertiefen, will ich kurz noch nach einem möglichen Ausweg aus diesem Dilemma fragen. Dieser müßte so beschaffen sein, daß die Geographiedidaktik, das Recht auf Freiheit der Forschung und das daraus ggf. entspringende auch geographische Forschungsinteresse einmal beiseite lassend, zumindest aus hochschulorganisatorischen Gründen einen unbeeinträchtigten Status und damit in der Forschung wie auch in der Lehre volle Entfaltungsmöglichkeiten hat. Mir scheint, eine solche Situation ist nur durch die Einrichtung vereinzelt ja bereits auch bestehender eigener geographiedidaktischer Institute zu erreichen. Wo dies aus personellen Gründen nicht möglich ist, müßte die Geographiedidaktik, in Anlehnung auch an HEURSEN (1984, S. 3-8), mit anderen, inhaltlich nahestehenden Fachdidaktiken, je nach eigenem Schwerpunkt entweder des sozial- und geisteswissenschaftlichen oder des naturwissenschaftlichen Bereichs, zu fachdidaktischen Instituten zusammengefaßt werden. Diese dann rein geographiedidaktischen oder gemischten fachdidaktischen Institute wären ihrerseits mit anderen fachdidaktischen Instituten zu fachdidaktischen Fachbereichen bzw. Fakultäten zusammenzufassen. Nur in einer solchen oder ähnlichen Organisationsstruktur wären alle Beteiligten frei von dem status- bzw. imagebedingten und entwicklungshemmenden Profilierungszwang, hätten sie statt dessen strukturell gleichwertige Voraussetzungen, Aufgaben und auch
Entwicklungschancen (vgl. auch кÖсK 21990). Ob diese hochschulorganisatorische Verselbständigung der Geographiedidaktik - wie der Fachdidaktiken überhaupt - allerdings auch aus hochschul- und speziell stellenpolitischer Sicht die beste Lösung ist, soll und kann hier nicht weiter untersucht werden. Eine enge Kooperation mit der Geographie müßte allerdings gesichert sein, und zwar vor allem im Blick auf die Lehre, je nach Situation jedoch auch hinsichtlich der Forschung (vgl. auch KÖCK ${ }^{2} 1986$, S. 128; SCHRAND 1981, S. 64/65; 1989, S. 7).

\section{Wissenschaftsgeschichtliche Begründung}

Wie stellt sich die Situation nun aus disziplingeschichtlicher Sicht dar?

Wenngleich es meines Wissens eine Geschichte der Geographiedidaktik, im Unterschied zu solchen des Geographieunterrichts wie auch der Geographie, bislang nicht gibt (vgl. auch SPERLING 1981, S. 107), so kann eine erste Antwort auf diese Frage doch auf der Grundlage des Aufkommens der geographiedidaktischen Fragestellung, manifestiert im Aufkommen entsprechender Publikationen, wie auch auf der Grundlage der Institutionalisierung der Geographiedidaktik in Hochschulen bzw. Universitäten versucht werden. Entsprechend ist hierbei, analog auch zur Geographie selbst, zu unterscheiden zwischen akademischer und vorakademischer Entwicklung. Angesichts der unzureichenden disziplingeschichtlichen Publikationslage haben die folgenden Ausführungen notwendig vorläufigen und teils hypothetischen Charakter.

Was die vorakademische Phase der Geographiedidaktik betrifft, so ist diese offenbar unabhängig von der Geographie entstanden. Denn folgt man ADELMANN (21962, S. 39), so fallen, sieht man von ersten mehr zufällig bestimmten Vorläufern erdkundlicher Unterweisung im Altertum sowie im Mittelalter ab (vgl. SCHRAND 1983, S. 77/78; SPERLING 1981, S. 99), die "Anfänge erdkundlicher Belehrungen in den Schulen während des 16 . Jahrhunderts ... in die Zeit eines verbalistischen Unterrichtsbetriebes. Ein höchst ungeographischer Betrieb war damals übliche Lehrweise, die sich auf Vortragen und Abfragen, auf geistloses Auswendiglernen eines topographischen Merkstoffes und auf mechanisches Aufzählen von Ländernamen mit Angabe der Flüsse und Meere, der Städte und Gebirge, der historischen und kulturgeschichtlichen Besonderheiten beschränkte.» Ähnlich charakterisiert SCHMIDT (51976, S. 11) die «Erdkunde der damaligen Zeit» als «eine bunte Sammlung aus Topographie, Geschichte, Religion, Statistik, aus Kuriositäten, Phantastereien und persönlichen Erlebnissen».

Vor dem Hintergrund dieser Unzulänglichkeit und Erbärmlichkeit des geographischen Unterrichts kam dann die geographiedidaktische Fragestellung 
auf, manifestiert in entsprechenden Publikationen, die die Behebung dieser Misere zum Ziel hatten. Bekanntlich war es COMENIUs, der im Rahmen seiner «Didactica magna» (1657) wohl als erster oder zumindest als einer der ersten Vorschläge zur inhaltlich-methodischen Verbesserung (auch) des Geographieunterrichts unterbreitete und hierdurch als erster oder zumindest als einer der ersten eine (auch) geographiedidaktische Publikation vorlegte, der im Jahre 1658 dann der "Orbis pictus» folgte, dessen Bilder als Anschauungsmittel zur Verdeutlichung geographischer Vorstellung dienten (ADELMANN 21962, S. 36, 39; SCHMIDT 51976 , S. 11). Auch GUTSMUTHS' "Versuch einer Methodik des geographischen Unterrichts» aus dem Jahre 1835 ging, um ein weiteres Beispiel anzusprechen, von der Unzulänglichkeit der geographischen Unterrichtspraxis aus. So ist der «Erste Abschnitt» seiner Methodik überschrieben mit «Einleitende Bemerkungen über die Unvollkommenheit des bis daher mehrentheils gangbaren Unterrichts in der Geographie», und dessen erstes Kapitel lautet: «Ursachen, welche die Vernachlässigung und das Fehlerhafte des geographischen Unterrichts bewirkt haben». Vor diesem Hintergrund breitete GUTSMUTHS dann seine eigenen Vorstellungen von Zielen, Inhalten, Mitteln und Wegen des Geographieunterrichts aus. Ähnlich mögen die meisten der frühen geographiedidaktischen Arbeiten angelegt sein, wovon SPERLINGS "Geographiedidaktische Quellenkunde» (1978, S. 524-548) für die Zeit von 1698 bis 180024 und für die Zeit von 1800 bis 1900116 Titel aufführt, und zwar nur selbständige deutschsprachige Gesamtdarstellungen des Geographieunterrichts.

Wenn ich die Genese der geographiedidaktischen Fragestellung wie der dementsprechenden ersten und frühen geographiedidaktischen Publikationen richtig deute, so vollzog sie sich unabhängig von der Geographie, und zwar in unmittelbarer Korrespondenz zu damals gegebener geographischer Unterrichtspraxis, und entspricht in ihrer Dialektik darüber hinaus offensichtlich dem Entsstehungsmuster didaktisch-methodischen Denkens überhaupt und generell. Danach setzt «didaktisches und methodisches Denken .. meistens als Kritik am Bestehenden ein. Fraglos anerkannte Ziele, Inhalte und Wege der in einem Gemeinwesen für den Unterricht Jugendlicher oder Erwachsener vorhandenen Einrichtungen werden infolge politischer, sozialer, kultureller oder innerpädagogischer Wandlungen fragwürdig. Häufig erwachsen aus solcher Kritik neue Vorschläge» (FISCHER Lexikon Pädagogik 1964, S. 50/51). Da didaktisches Fragen als solches und im wesentlichen fachunabhängig aber bereits seit der Antike besteht (vgl. EBINGER 1971, S. 19; FISCHER Lexikon Pädagogik 1964, S. 50), kann man das geographiedidaktische Fragen seit dem 17. Jahrhundert dann als einen Fall von fach- bzw. sachgebiets- bezogener Ausdifferenzierung des didaktischen Fragens überhaupt verstehen, was zugleich konsistent ist zu der vorhin vorgenommenen disziplinsystematischen Zuordnung der Geographiedidaktik zur Didaktik.

Betrachtet man im Vergleich dazu nun die akademische Disziplingeschichte, so beginnt diese bekanntlich erst um 1960. Bis dahin und auch noch darüber hinaus spielte die Geographiedidaktik, wie jede Fachdidaktik, in der gymnasialen Lehrerbildung überhaupt keine Rolle, und in der Volksschullehrerbildung war sie im Rahmen der seminaristischen Lehrerbildung des 19 . und frühen 20. Jahrhunderts sowie in den ab den 20er Jahren aufkommenden Pädagogischen Akademien wie zunächst auch noch in den nach 1945 entstehenden Pädagogischen Hochschulen im Rahmen der Allgemeinen Didaktik bzw. Pädagogik allenfalls mitvertreten. Als selbständige Disziplin an wissenschaftlichen Hochschulen einschließlich später der Universitäten etablierte sich die Geographiedidaktik, parallel zu den übrigen Fachdidaktiken, jedoch erst ab den $60 \mathrm{er}$ Jahren vor allem im Gefolge der Pädagogischen Hochschultage von 1959 (Tübingen) und 1962 (Trier). Dabei geschah auch dies weitgehend unabhängig von der Geographie, eher sogar gegen deren Willen, sofern nicht stellenpolitische Überlegungen dafür sprachen, in keinem Fall aber durch eine Art Zellteilung der Geographie. Vielmehr scheint dies ein Ergebnis des hochschul- und wissenschaftspolitischen Drängens der Pädagogischen Hochschulen und speziell der Pädagogik und Didaktik wie auch der aufkommenden Fachdidaktiken selbst zu sein und kann insoweit wohl ebenfalls wieder als fachbezogene Ausdifferenzierung der Allgemeinen Didaktik bzw. Pädagogik verstanden werden (vgl. EBINGER 1971, S. 20; FISCHER Lexikon Pädagogik 1964 , S. $188-194$; отто 1984 , S. 22-23, 31, 41). Auch dies ist dann konsistent zu der vorhin vorgenommenen disziplinsystematischen Zuordnung der Geographiedidaktik zur Didaktik.

Wie diese kurzen, wenngleich noch nicht hinreichend abgesicherten Ausführungen nun zeigen, scheint die Geographiedidaktik auch aus disziplingeschichtlicher Sicht kein Ableger bzw. Anhängsel der Geographie, sondern Produkt der sukzessiven Weiterentwicklung und speziell Ausdifferenzierung der Allgemeinen Didaktik bzw. Pädagogik zu sein, wobei diese sich in Korrespondenz zu den entsprechenden Unterrichtsfächern als den jeweiligen Forschungsgegenständen vollzog. Wohl allerdings erfolgte die Entwicklung der Geographiedidaktik, wie auch des Geographieunterrichts selbst, in enger Wechselwirkung auch zur Geographie, was dann bedeutet, daß sowohl die Geographiedidaktik durch Konzepte, Entwicklungen, Erkenntnisfortschritte usw. in der Geographie angeregt und beeinflußt wurde als auch die Geographie von solchen der 
Geographiedidaktik (vgl. ADELMANN 21962, S. 31-36; SCHRAND 1983, S. 90/91).

Wenn die Geographiedidaktik nun, wie diese Ausführungen gezeigt haben, eine Wissenschaft aus eigenem Recht, eine Wissenschaft wie jede andere also ist, dann gibt dies zunächst Anlaß zu Selbstbewußtsein statt zu Minderwertigkeitskomplexen. Zugleich aber bedeutet dies auch Anspruch, und zwar dann eben auch diejenige Leistung zu erbringen, die man üblicherweise und zu Recht von einer Wissenschaft erwartet!

\section{Literatur}

ABLER, R./ADAMS, J. S./GOULD, P. (1972): Spatial organization. - London u. a.

ADELMANN, J. (21982): Methodik des Erdkundeunterrichts. - München.

BAUER, L. (1974): Curriculum und Fachdidaktik. - In: KREUZER, G. et al. (Hrsg. 1974): Didaktik der Geographie in der Universität. München. S. 9-27.

BIRKENHAUER, J. (1975): Zur Fachdidaktik als Unterrichtswissenschaft zwischen Erziehungswissenschaft und Fachwissenschaft. - In: PAPKE, K. et al. (Bearbeiter 1975): Unterrichtswissenschaften und integrierte Lehrerbildung an $\mathrm{Ge}-$ samthochschulen. Berlin. S. 160-176.

BIRKENHAUER, J. (1977): Geographische Fachdidaktik im Spannungsfeld von Wissenschaft und Pädagogik. - In: REINHARDT, K. H. (Hrsg. 1977): Die Geographie und ihre Didaktik zwischen Umbruch und Konsolidierung. Frankfurt. S. $146-158$.

BIRKENHAUER, J. (1980): Stellung der Fachdidaktik zwischen Erziehungswissenschaft und Fachwissenschaft. - In: KREUZER, G. (Hrsg. 1980): Didaktik des Geographieunterrichtes. Hannover u. a. S. 13-22.

BÖNSCH, M. (1985): Das Verhältnis von Theorie und Praxis in der Allgemeinen Didaktik. - In: SCHWEIZER SCHULE. S. 2-8.

DÜRR, H. (1979): Für eine offene Geographie, gegen eine Geographie im Elfenbeinturm. - Karlsruhe.

EBINGER, H. (1971): Einführung in die Didaktik der Geographie. - Freiburg.

EBINGER, H. (1977): Didaktik der Geographie an der Universität. - Das Hamburger Beispiel - In: REINHARDT, K. H. (Hrsg. 1977): Die Geographie und ihre Didaktik zwischen Umbruch und Konsolidierung. Frankfurt. S. 159-172.

FISCHER LEXIKON PÄDAGOGIK (1964). - Frankfurt.

GUTSMUTHS, J. Chr. Fr. von (1835): Versuch einer Methodik des geographischen Unterrichts. - Weimar. (Nachdruck: Trier 1984).

HARD, G. (21975): Bibliographie Raisonnée zur Fachdidaktik. - In: BARTELS, D./HARD, G. (21975): Lotsenbuch für das Studium der Geographie als Lehrfach. Bonn u. a. S. 399-451.

HARD, G. (1979): Die Disziplin der Weißwäscher. Über Genese und Funktionen des Opportunismus in der Geographie. - In: SEDLACEK, P. (Hrsg. 1979): Zur Situation der deutschen Geographie zehn Jahre nach Kiel. Osnabrück. S. 11-44.
HEURSEN, G. (1984): Didaktik im Umbruch: Fachdidaktik auf dem Weg zu ihrer Eigenständigkeit. - In: HEURSEN, G. (Hrsg. 1984): Didaktik im Umbruch. Königstein. S. 1-21.

HOLZKAMP, K. (1968): Wissenschaft als Handlung. - Berlin.

JAHN, W. (1974): Integration als didaktisches Axiom geographischer Wissenschaft und Lehre. - In: KREUZER, G. et al. (Hrsg. 1974): Didaktik der Geographie in der Universität. München. S. 62-68.

KÖCK, H. (1986.1): Zur methodologischen Grundlegung der Geographiedidaktik. - In: HUSA, K.NIELHABER, Chr./ WOHLSCHLÄGL, H. (Hrsg. 1986): Beiträge zur Didaktik der Geographie. Festschrift Ernest Troger zum 60. Geburtstag. Bd. 2. Wien S. 19-46.

KÖCK, H. (1986.2): Die Geographiedidaktik als Wissenschaft - eine Skizze ihres methodologischen Grundrisses. In: GEOGRAPHIE UND IHRE DIDAKTIK. S. 113-133.

KÖCK, H. (1986.3): Ziele und Inhalte (des Geographieunterrichts). - In: KÖCK, H. (Hrsg. 1986): Grundlagen des Geographieunterrichts. Köln. S. 129-130, 137-208, 316-328.

KÖCK, H. (1987): Räumliche Ordnung - universale und geographische Kategorie. - In: KÖCK, H. (Hrsg. 1987): Mensch und Raum - Paul Schäfer zum 65. Geburtstag gewidmet. Hildesheim u. a. S. 31-48.

KÖCK, H. (1988.1): Zu einigen Grundfragen von Geographieunterricht und Geographiedidaktik - Stellungnahme zu den Diskussionsbeiträgen von Josef Birkenhauer unter Einbezug desjenigen von Hartmut Voigt. - In: GEOGRAPHIE UND IHRE DIDAKTIK. S. 86-95, 135-147.

KÖCK, H. (1988.2): Geographie und Geographiedidaktik ein methodologischer Statusvergleich. - In: GEOGRAPHISCHE RUNDSCHAU. S. 54-56, 58.

KÖCK, H. (1989): Aufgabe und Aufbau des Geographieunterrichts. - In: GEOGRAPHIE UND SCHULE. 57. S. 11-25.

KÖCK, H. (1990.1): Geographiedidaktik - disziplinsystematischer Standort und wissenschaftliche Wertigkeit. - In: KECK, R. W. et al. (Hrsg. 1990): Fachdidaktik zwischen Allgemeiner Didaktik und Fachwissenschaft. Bad Heilbrunn. Im Druck.

KÖCK, H. (1990.2): Eine Wissenschaft auf der Suche nach ihrer Identität - Gedanken zum Selbstverständnis der Geographiedidaktik. In: Kontinuität und Innovationen in der Geographiedidaktik. Festkolloquium Josef Birkenhauer zum 60. Geburtstag, München, im Druck.

KUHN, Th. S. (1962/73): Die Struktur wissenschaftlicher Revolutionen. - Frankfurt.

MENNE, A. (1980): Einführung in die Methodologie. - Darmstadt.

OBLINGER, H. (1974): Über die Funktion der Fachdidaktik zwischen Erziehungswissenschaft und Fachwissenschaft. In: KREUZER, G. et al. (Hrsg. 1974): Didaktik der Geographie in der Universität. München S. 89-95.

OTTO, G. (1984): Zur Etablierung der Didaktiken als Wissenschaft. - In: HEURSEN, G. (Hrsg. 1984): Didaktik im Umbruch. Königstein. S. 22-58.

PÖPPEL, K. G. (1987): Schulgeographie - ein Fall von Kulturpädagogik? - In: KÖCK, H. (Hrsg. 1987): Mensch und Raum - Paul Schäfer zum 65. Geburtstag gewidmet. Hildesheim u. a. S. 18-30.

POPPER, K. R. (1984.8): Logik der Forschung. - Tübingen.

RICHTER, D./SCHULTZE, A./SCHRETTENBRUNNER, $\mathrm{H}$. (1971): Wege zu veränderten Bildungszielen im Schulfach 
"Erdkunde". - In: GEOGRAPHISCHE RUNDSCHAU. S. $146-149$.

RIEDL, R. (1980.2): Biologie der Erkenntnis. - Berlin u. a.

RIEDL, R. (1985): Die Stellung des Weltbildes. Biologische Grundlagen des Erklärens und Verstehens. - Berlin u. a.

SCHMIDT, A. (1976.5): Der Erdkundenunterricht. - Bad Heilbrunn.

SCHMIEL, M. (1978): Einführung in fachdidaktisches Denken. - München.

SCHRAND, H. (1981): Zur Stellung der Fachdidaktik in der universitären Lehrerausbildung. - In: GEOGRAPHIE UND IHRE DIDAKTIK. S. 58-65.

SCHRAND, H. (1983): Zur Geschichte der Geographie in Schule und Hochschule. - In: MANNZMANN, A. (Hrsg. 1983): Geschichte der Unterrichtsfächer II, München. S. 74-107.

SCHRAND, H. (1989): Zur Lage der Geographiedidaktik Ende der 80er Jahre. - In: GEOGRAPHIE UND SCHULE. 57. S. 2-11.
SCHREIBER, Th. (1981): Kompendium Didaktik Geographie. - München.

SEIFFERT, H. (101983): Einführung in die Wissenschaftstheorie. Bd. 1. - München.

SPERLING, W. (1969): Stellung und Aufgaben der Didaktik der Geographie im System der geographischen Wissenschaft und im Verhältnis zur Angewandten Geographie. - In: GEOGRAPHISCHE RUNDSCHAU. S. 81-88.

SPERLING, W. (1978): Geographiedidaktische Quellenkunde. - Duisburg.

SPERLING, W. (1981): Geschichte des Geographieunterrichts und der Geographiedidaktik zwischen Geographiegeschichte und Bildungsgeschichte. - In: SPERLING, W. (Hrsg. 1981): Theorie und Geschichte des geographischen Unterrichts. Braunschweig. S. 96-117.

STEUBER, H. (1981): Grundlagen der Methodologie der Didaktik. - Frankfurt.

VOLLMER, G. (1975): Evolutionäre Erkenntnistheorie. Stuttgart.

WEINGARTNER, P. (21978): Wissenschaftstheorie. Bd. 1. Stuttgart-Bad Cannstatt. 